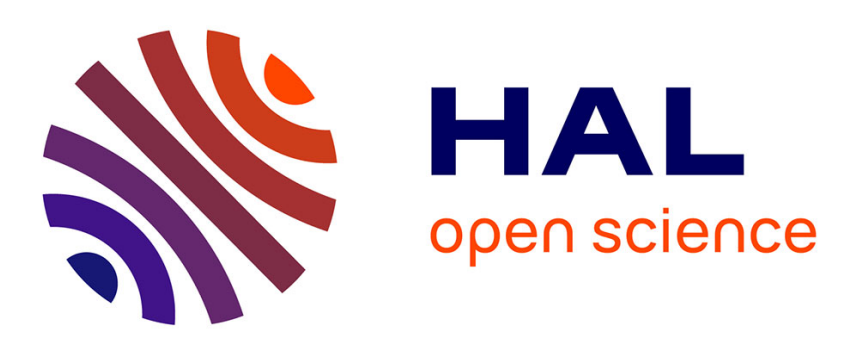

\title{
Deep Learning-based Signal Detection for Uplink in LoRa-like Networks
}

Angesom Ataklity Tesfay, Eric Pierre Simon, Sofiane Kharbech, Laurent Clavier

\section{- To cite this version:}

Angesom Ataklity Tesfay, Eric Pierre Simon, Sofiane Kharbech, Laurent Clavier. Deep Learning-based Signal Detection for Uplink in LoRa-like Networks. IEEE 32nd Annual International Symposium on Personal, Indoor and Mobile Radio Communications, PIMRC 2021, Sep 2021, Helsinki, Finland. pp.617-621. hal-03373813

\section{HAL Id: hal-03373813 https://hal.science/hal-03373813}

Submitted on 11 Oct 2021

HAL is a multi-disciplinary open access archive for the deposit and dissemination of scientific research documents, whether they are published or not. The documents may come from teaching and research institutions in France or abroad, or from public or private research centers.
L'archive ouverte pluridisciplinaire HAL, est destinée au dépôt et à la diffusion de documents scientifiques de niveau recherche, publiés ou non, émanant des établissements d'enseignement et de recherche français ou étrangers, des laboratoires publics ou privés. 
This article has been accepted for publication in IEEE 32nd Annual International Symposium on Personal, Indoor and Mobile Radio Communications, but has not been fully edited. Content may change prior to final publication. Citation information: A. A. Tesfay, E. P. Simon, S. Kharbech, and L. Clavier, "Deep Learning-Based signal detection for uplink in LoRa-Like networks," in 2021 IEEE 32nd PIMRC, Helsinki, Finland, Sep. 2021.

\title{
Deep Learning-based Signal Detection for Uplink in LoRa-like Networks
}

\author{
Angesom Ataklity Tesfay ${ }^{1}$, Eric Pierre Simon ${ }^{1}$, Sofiane Kharbech ${ }^{2}$, and Laurent Clavier ${ }^{1,2}$ \\ ${ }^{1}$ Univ. Lille, CNRS, UMR 8520 - IEMN, F-59000, Lille, France (e-mail: firstname.name@ univ-lille.fr) \\ ${ }^{2}$ IMT Lille Douai, France (e-mail: firstname.name@imt-lille-douai.fr)
}

\begin{abstract}
The increasing number of devices together with uncoordinated transmissions result in a major challenge of scalability in the Internet of things. This paper deals with signal detection in the uplink of a LoRa network through a deep learning-based approach. Two strategies are proposed: regression for bit detection based on a deep feedforward neural network and classification for symbol detection based on a convolutional neural network. These receivers can decode a selected user's signals when multiple users simultaneously transmit over the same frequency band with the same spreading factor. Simulation results show that both receivers outperform the classical LoRa one in the presence of interference. The results show that the introduced approach is relevant to deal with the scalability issue.

Index Terms-LoRa, IoT, deep learning, neural networks, capture effect.
\end{abstract}

\section{INTRODUCTION}

In the next few years, the number of connected Internet of Things (IoT) devices is expected to grow exponentially, which requires technology that can handle large-scale connectivity. In recent years, Low-Power Wide Area Network (LPWAN) technologies, such as LoRa and SigFox in the license-free frequency band, have been introduced to deal with this issue. LoRa uses a Chirp-Spread Spectrum Modulation scheme. Different spreading factors (SF) and bandwidth settings can be used to achieve quasi-orthogonal transmissions. However, when two or more devices simultaneously transmit with the same SF on the same frequency band, collisions occur at the receiver [1], generally resulting in packet loss. In fact, sometimes, one packet can be correctly decoded despite the collision. This is the capture effect. This can be achieved when the desired signal is sufficiently stronger than the interfering one. Such results have been presented in LoRa communications in [2]. However, an interleaved chirp spreading (ICS) modulation scheme is used, and this is not directly backward compatible with the LoRaWAN standard. This principle is also used in serial interference cancellation schemes. In [3], the strongest user can be decoded, then suppressed, and the second strongest can, in turn, be decoded. The capture effect is also used in [4], where two superposed signals, synchronized or slightly desynchronized, can be decoded using timing information. However, ensuring synchronization is not realistic in the uplink of wide-area networks considered in this paper.

Nevertheless, the capture effect requires a sufficient power gap between the desired and the interfering signal. In this paper, we propose two signal detection schemes for the uplink in LoRa-like networks based on deep learning that significantly improve the receiver's ability to accurately retrieve a signal, even when interfering users are present.

In the literature, some works employ the deep learning approach for IoT networks [5], [6], but not for detection. To our knowledge, there are no works yet that investigate detection in that context using deep learning.

The contribution of this paper is three-fold: (i) Designing two new receivers based on deep learning for detection in LoRa networks. The first one deals with bit detection with a regression approach, as in [7] for the OFDM context, and the second one deals with symbol detection with a classification approach. These receivers can cope with interference coming from other LoRa users, and both strongly improve the capture effect compared to the classical LoRa receiver; (ii) It is shown that, once they have information on the power levels, the designed receivers are further efficient, mainly if the interfering users are stronger than the user to be decoded; and (iii) The outstanding performance of the new receivers is achieved while maintaining an efficient complexity order.

The rest of the paper is organized as follows. Section II describes the system model. Section III presents the proposed deep learning-based receivers. Section IV shows simulation results and discussions. Conclusions are drawn in Section V.

\section{SySTEM MODEL}

In LoRa, each symbol carries SF bits, representing $M=2^{\mathrm{SF}}$ possible values. The signal bandwidth is denoted by $B$, and the symbol duration is $T_{s}=M T$, where $T=1 / B$. The SF ranges from 7 to 12 . Each LoRa symbol, also called chirp, consists of a linear frequency shift over $T_{s}$. It is built from a raw chirp $c(t)$. The raw chirp has the instantaneous frequency $\frac{B}{T_{s}} t$, yielding the base-band expression [8]:

$$
c(t)=\exp \left(2 \imath \pi \frac{B}{2 T_{s}} t^{2}\right) \quad t \in\left[-\frac{T_{s}}{2}, \frac{T_{s}}{2}\right] .
$$

Let $Q$ be the number of transmitted LoRa symbols in a packet. The transmitted symbol of the $j$ th user at time $q T_{s}$ $(q=0, \cdots, Q-1)$ is $m_{j}^{(q)} \in\{0, \ldots, M-1\}$. This symbol is carried by performing a cyclic shift of the raw chirp by $\delta_{j}^{(q)}=m_{j}^{(q)} T$, as illustrated in Fig. 1 Hence, the coded chirp 
This article has been accepted for publication in IEEE 32nd Annual International Symposium on Personal, Indoor and Mobile Radio Communications, but has not been fully edited. Content may change prior to final publication. Citation information: A. A. Tesfay, E. P. Simon, S. Kharbech, and L. Clavier, "Deep Learning-Based signal detection for uplink in LoRa-Like networks," in 2021 IEEE 32nd PIMRC, Helsinki, Finland, Sep. 2021.

is:

$$
s_{j}^{(q)}(t)=\left\{\begin{array}{c}
\exp \left(2 \imath \pi\left[\frac{B}{2 T_{s}} t^{2}+\frac{m_{j}^{(q)}}{T_{s}} t\right]\right), \\
t \in\left[-\frac{T_{s}}{2}, \frac{T_{s}}{2}-\delta_{j}^{(q)}\right), \\
\exp \left(2 \imath \pi\left[\frac{B}{2 T_{s}} t^{2}+\left(\frac{m_{j}^{(q)}}{T_{s}}-B\right) t\right]\right) \\
t \in\left[\frac{T_{s}}{2}-\delta_{j}^{(q)}, \frac{T_{s}}{2}\right] .
\end{array}\right.
$$

The transmitted signal of user $j, s_{j}(t)$, is then $s_{j}(t)=$ $\sum_{q=0}^{Q-1} s_{j}^{(q)}\left(t-q T_{s}\right)$. The transmitted LoRa packet is obtained by adding a preamble composed of raw chirps.

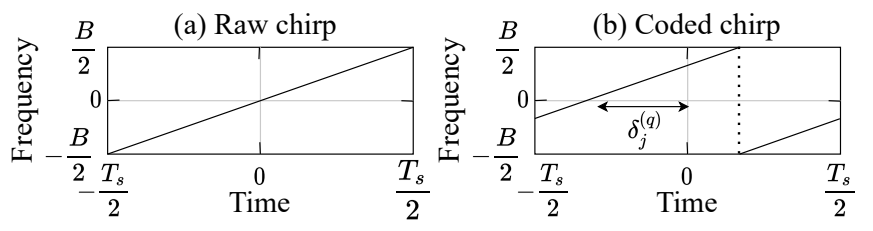

Fig. 1: (a) Raw chirp (b) Coded chirp associated with $m_{j}^{(q)}$.

A circle of radius $r_{\max }$ with one gateway located at the center is considered, including a user free guard-zone around the gateway with radius $r_{\text {min }}$. We assume $N_{i}$ interfering users having the same SF. $N_{i}$ is randomly drawn from a Poisson distribution with parameter $\lambda$. The $2 \mathrm{D}$ coordinates of the $N_{i}$ interfering users are uniformly distributed while considering only positions within the disc defined by $r_{\max }$ and $r_{\min }$. Since different devices operate autonomously, the transmission between nodes is asynchronous.

The synchronization is carried out as follows: a correlation is first performed between the received signal and the preamble. Then the highest peak of the correlation is selected, and the received signal is synchronized on it. The user corresponding to this peak is the desired user to be decoded, indexed by $j$ here. The received signal associated with symbol $q$ of user $j$ sampled at $t=n T, n=-\frac{M}{2}, \cdots, \frac{M}{2}-1$ is:

$$
r^{(q)}[n]=h_{j} s_{j}^{(q)}[n]+\sum_{i \in \mathcal{I}} h_{i} s_{j, i}^{\text {interf, }(q)}[n]+w^{(q]}[n],
$$

where $s_{j}^{(q)}[n]=s_{j}^{(q)}(n T), \mathcal{I}$ is the set of interfering users $\left(|\mathcal{I}|=N_{i}\right)$ and $w_{q}[n] \sim \mathcal{C N}\left(0, \sigma^{2}\right)$ is a circularly symmetrical complex Gaussian noise. $h_{j}$ and $h_{i}$ are the channel coefficients for users $j$ and $i$, respectively.

The collision between the $q$ th symbol of the selected user $j$ and two consecutive symbols of an interfering user $i$ is illustrated in Fig. 2. The interfering part of user $i$ is denoted by $s_{j, i}^{\text {interf, }(q)}$. The delay between user $j$ and user $i$ (accounting for asynchronous transmissions between the nodes) is $\tau_{j, i}$.

To demodulate, the classical LoRa receiver first multiplies the samples of the received signal by the conjugate of the raw chirp, yielding $y^{(q)}[n]=r^{(q)}[n] c^{*}[n]$, where $c^{*}[n]=c^{*}(n T)$. Then, a Fast Fourier Transform (FFT) is applied:

$$
Y^{(q)}[k]=\sum_{n=-M / 2}^{M / 2} y^{(q)}[n] e^{-2 \imath \pi \frac{n k}{M}}, k=0, \ldots, M-1 .
$$

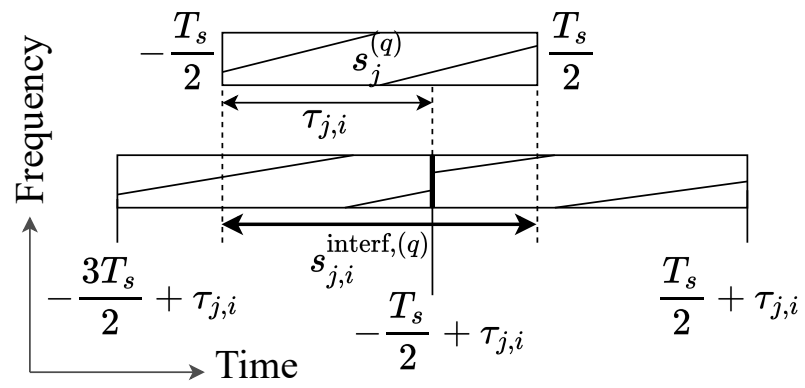

Fig. 2: Collision between a symbol of desired user and two consecutive symbols of the interfering user.

In the classical LoRa receiver, the symbol $m_{j}^{(q)}$ is estimated by searching for the frequency index where the modulus of (4) is maximum. However, collisions occur when multiple users transmit simultaneously using the same SF, yielding possible detection errors.

\section{Proposed DeEp LeARning-BASEd ReCEIVER}

This section proposes two receivers: the first is based on Deep Feedforward Neural Network (DFNN), and the second on Convolutional Neural Network (CNN).

\section{A. Deep Feedforward Neural Network-based receiver}

As indicated in Fig. 3, the detector relies on a DFNN architecture with four hidden layers. The number of nodes in each hidden layer is $8 M, 4 M, 2 M$, and $M$. The input is the modulus of the de-chirped received samples after the FFT (4), yielding $M$ input nodes. The output is the bits of the transmitted symbol, yielding SF output nodes. The ReLU function is used as the activation function in the hidden layers. The sigmoid function is applied to map the outputs to the interval $[0,1]$ in the output layer. Batch normalization $(\mathrm{BN})$ is embedded in the hidden layers to prevent overfitting.

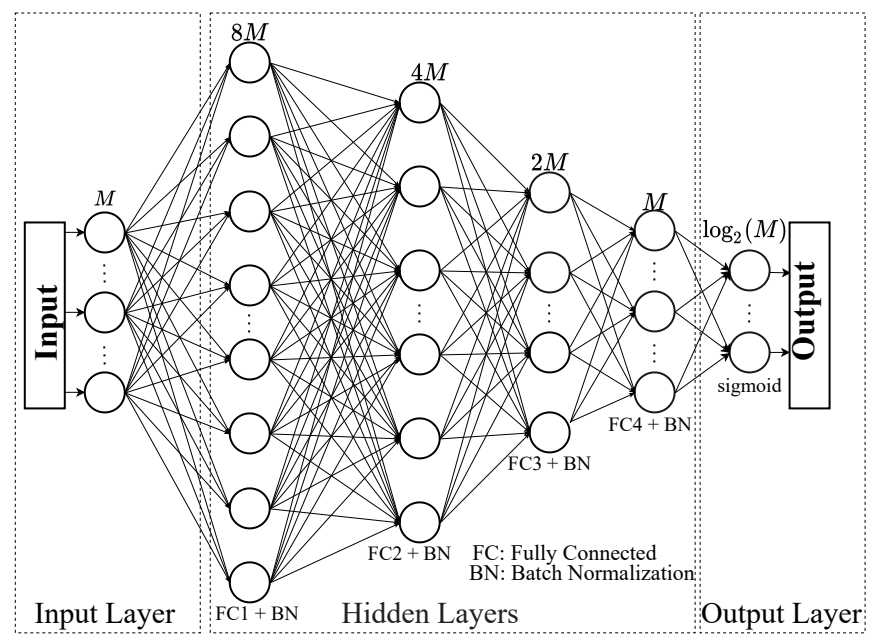

Fig. 3: Deep Feedforward Neural Network architecture.

The DFNN is trained so that the error between the outputs and the transmitted bits is minimized. The error is evaluated through the $L_{2}$ mean squared error function. Then, the detected 
This article has been accepted for publication in IEEE 32nd Annual International Symposium on Personal, Indoor and Mobile Radio Communications, but has not been fully edited. Content may change prior to final publication. Citation information: A. A. Tesfay, E. P. Simon, S. Kharbech, and L. Clavier, "Deep Learning-Based signal detection for uplink in LoRa-Like networks," in 2021 IEEE 32nd PIMRC, Helsinki, Finland, Sep. 2021.

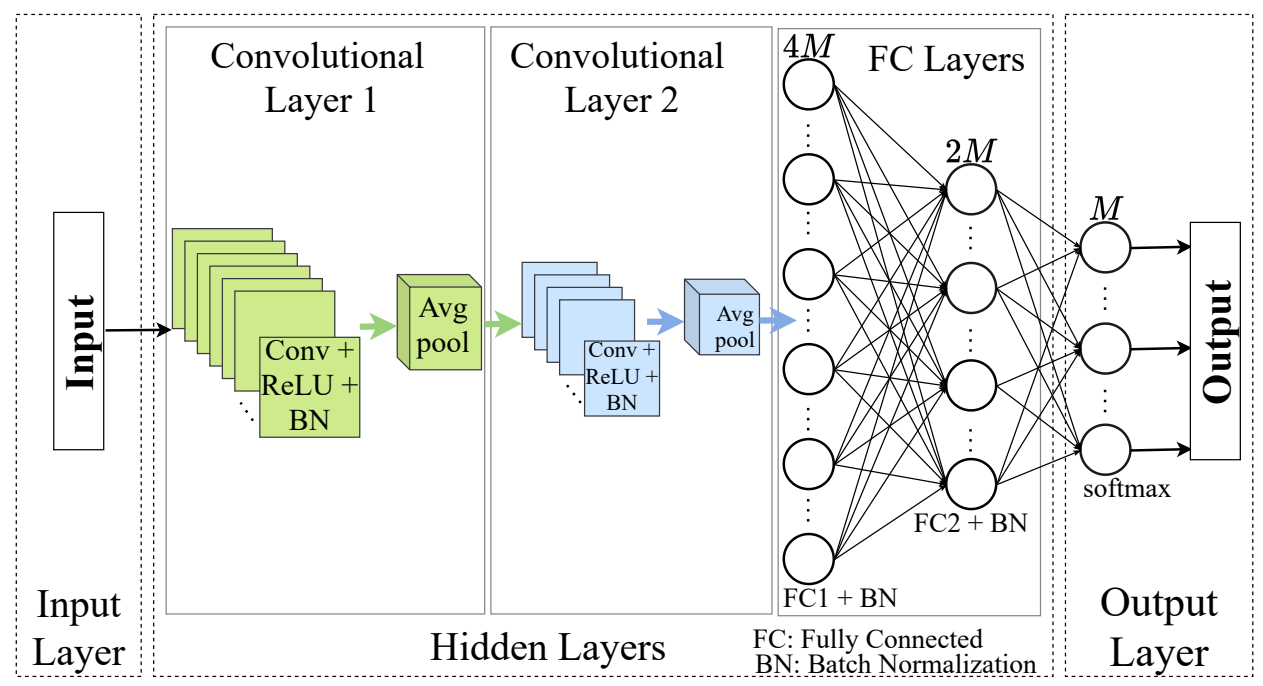

Fig. 4: Convolutional Neural Network architecture.

bits are obtained by thresholding the outputs. For the sigmoid function, the corresponding threshold is 0.5 .

\section{B. Convolutional Neural Network-based receiver}

Differently from the common feedforward architecture, CNN relies mainly on convolution operations within the socalled convolutional layers. For this architecture, the input is presented as an $M \times M$ binary image containing the modulus plots of (4), as illustrated in Fig. 5. The $M$ nodes at the output layer correspond to the $M$ symbols to be detected. Here, we use a structure that includes two convolutional layers and two fully connected layers (cf. Fig. 4). We set $M / 4$ and

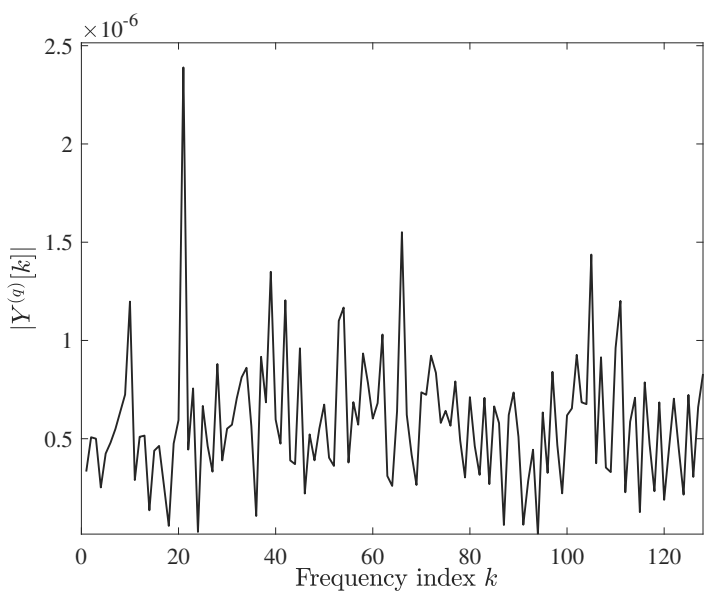

Fig. 5: Plot of $\left|Y^{(q)}[k]\right|(k=0, \cdots, M-1)$ for a symbol of $m^{(q)}=$ 20 with $\mathrm{SNR}=-10 \mathrm{~dB}$ and $\mathrm{SF}=7$.

$M / 2$ kernels for the first and second convolutional layers, respectively. The kernel size is set to $4 \times 4$ for both layers. A pooling layer follows convolution steps to reduce the feature map's dimension while keeping the most relevant information. The filter used for the average pooling layer is of size $2 \times 2$, and the stride is 2 . The output of the second pooling layer is flattened to be the input of the fully connected layer. The first fully connected layer has $4 M$ nodes and the second one has $2 M$ nodes. Similarly to the DFNN, the ReLU function is used as the activation function, and batch normalization is performed. For the output classification layer, we employ the softmax function. The CNN is trained to minimize the crossentropy loss between the output and the transmitted symbols.

\section{Simulation Results}

In this section, we provide simulation results to evaluate the proposed DFNN and CNN-based receivers' performance. All the parameters of the neural network are set through a hyperparameter tuning process. Training is done singly offline using training data generated from simulation for each SNR value. For the LoRa modulation, the bandwidth $B=250 \mathrm{kHz}$ is considered, with $\mathrm{SF}=7$, yielding $M=128 . r_{\max }$ and $r_{\text {min }}$ are set to $1 \mathrm{~km}$ and $200 \mathrm{~m}$, respectively. In LoRa, the devices are usually located far from the gateway since LoRa is designed for long range communications. The proposed detectors are compared with the classical LoRa detector in terms of symbol error rate (SER).

In the following, first, we present results where $N_{i}$ is a random variable. Then, in order to investigate the capture effect, the number of interfering users is forced to one $\left(N_{i}=1\right)$. Capture effect is the receiver's ability to decode one out of two (or more) colliding users correctly, usually the strongest one but not necessarily with the proposed receiver.

\section{A. Results for $N_{i} \sim \mathcal{P}(\lambda)$}

As described in section II the total number of interfering users $N_{i}$, in the time interval of interest, is drawn from a Poisson distribution, i.e., $N_{i} \sim \mathcal{P}(\lambda)$, with the following values for $\lambda=0.25,0.5,0.7$, and 1 . Training and test are done for each $(\mathrm{SNR}, \lambda)$ couple. Note that LoRa receivers can estimate the SNR. Results are presented in Fig. 6 As a lower bound, the case when there is no interfering user, i.e., $\lambda=0$, is considered (dashed line curves). It is shown that the proposed 


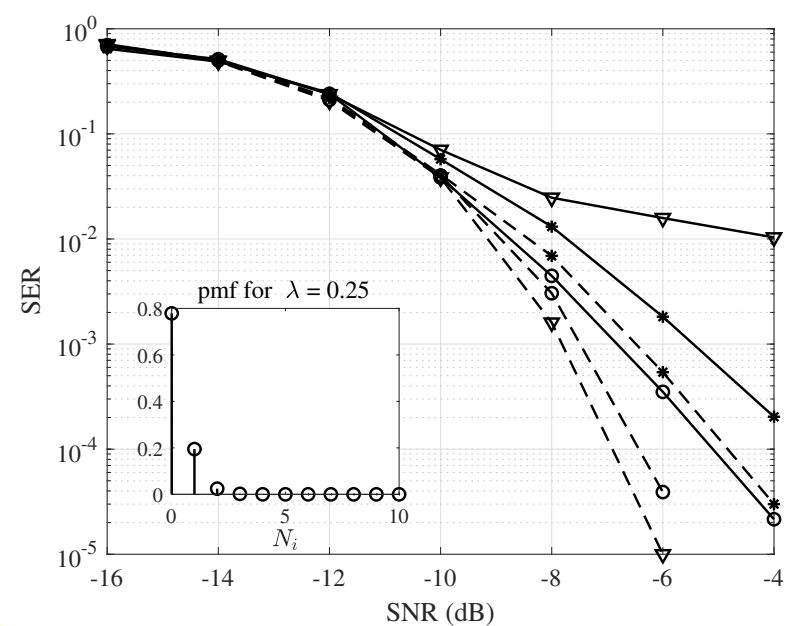

(a) $\lambda=0.25$

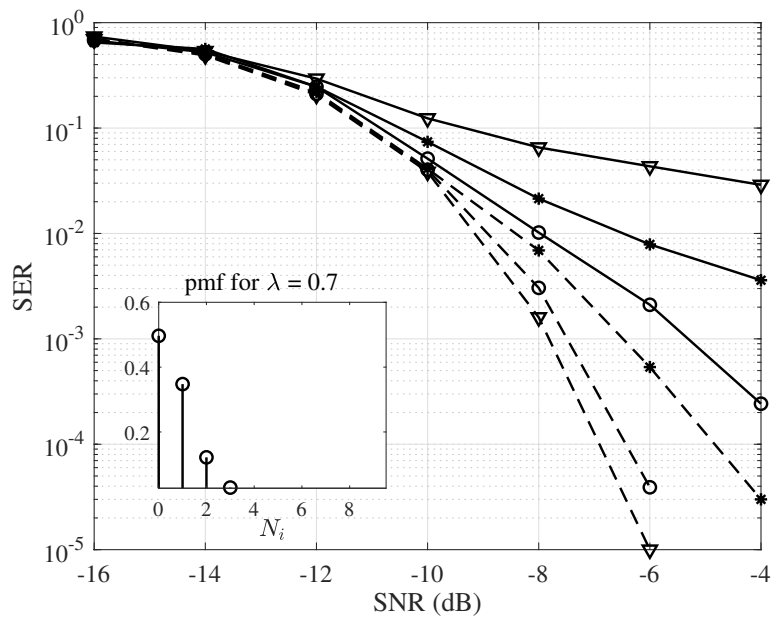

(c) $\lambda=0.7$

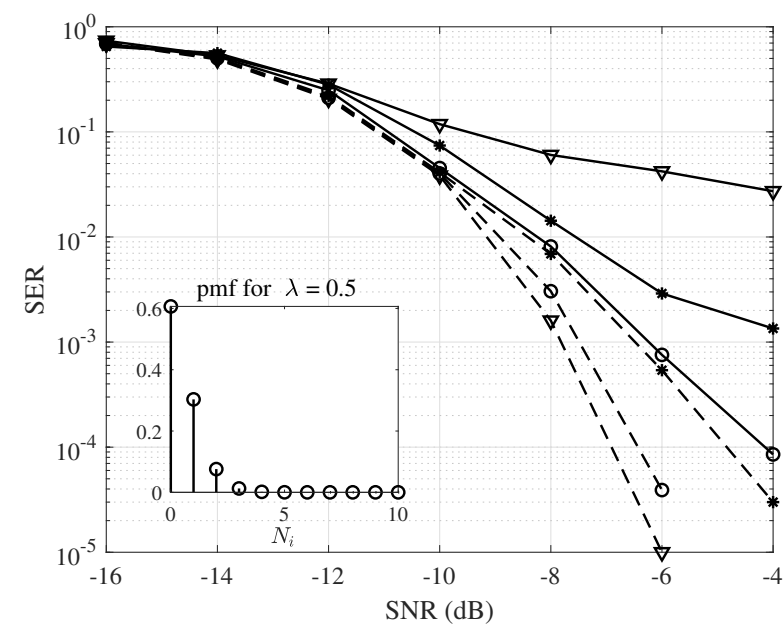

(b) $\lambda=0.5$

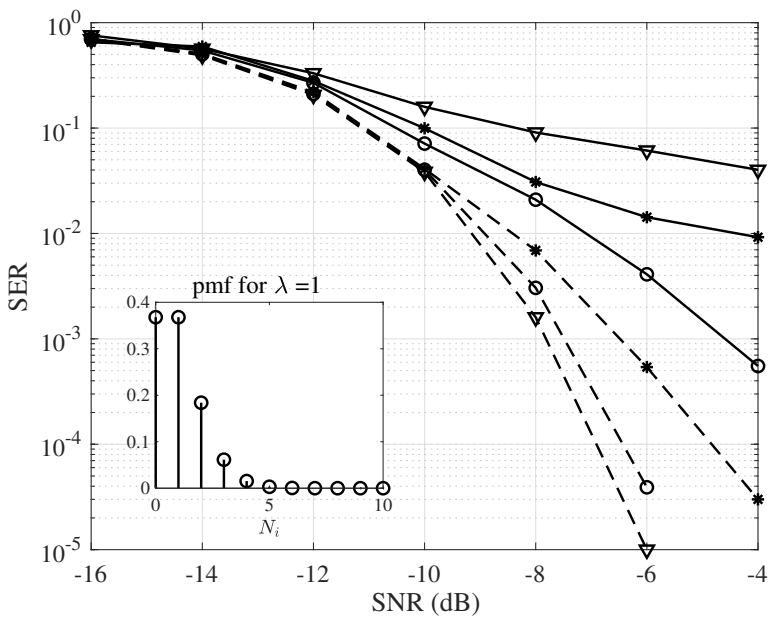

(d) $\lambda=1$

Fig. 6: Symbol error rate as a function of the SNR for different detection approaches: classical decoder [ $\nabla$ ], DFNN-based [*], and CNN-based [o]. For all figures, two scenarios are considered: no interference (plots in dashed line [--]) and when interference can happen (plots in solid line [-]). The plot inside show the probability mass function (pmf) related to the number of interfering users.

receivers outperform the classical LoRa one for $\lambda \neq 0$. For $\lambda=0$, the SER of the CNN is close to that of the classical LoRa receiver, the optimal in that case, whereas the DFNN is not as efficient with a $2 \mathrm{~dB}$ penalty for a target SER of $10^{-4}$. For the lowest number of interfering users $(\lambda=0.25$ and 0.5), the SER of the two proposed receivers is in the order of $10^{-3}$ for SNR $=-7.5 \mathrm{~dB}$ (cf. Figs. 6a and $6 \mathrm{~b}$. For the same SNR value, in the case of the higher number of interfering users $(\lambda=0.7)$, the SER of the CNN-based receiver remains in the order of $10^{-3}$ whereas the SER of the other receivers degrades (cf. Fig. 6c and 6d). Besides, as the number of interfering users increases, the classical LoRa receiver endures higher performance loss than the $\mathrm{CNN}$-based receiver.

\section{B. Capture Effect $-N_{i}=1$}

The goal is now to analyze the impact of one interfering user with different levels of signal-to-interference ratio (SIR). This is done by generating a new test set for which $N_{i}$ is forced to one and testing the previously trained networks with this specific test set. The one that was trained for the $(\lambda=0.25$, $\mathrm{SNR}=-6 \mathrm{~dB}$ ) couple is considered. Fig. 7 plots the obtained SER as a function of the SIR (solid line).

Additionally, as a perspective, we also investigate what would happen if the SIR could be estimated at the receiver. To do so, we do a new training of the networks for the different (SIR, SNR $=-6 \mathrm{~dB}$ ) couples with $N_{i}=1$, and we use the appropriate network for the test. Clearly, in this context, the deep learning-based approaches outperform the classical receiver for $\operatorname{SIR} \leq 4 \mathrm{~dB}$.

When SIR $>0 \mathrm{~dB}$, that is, the selected user's signal is the 


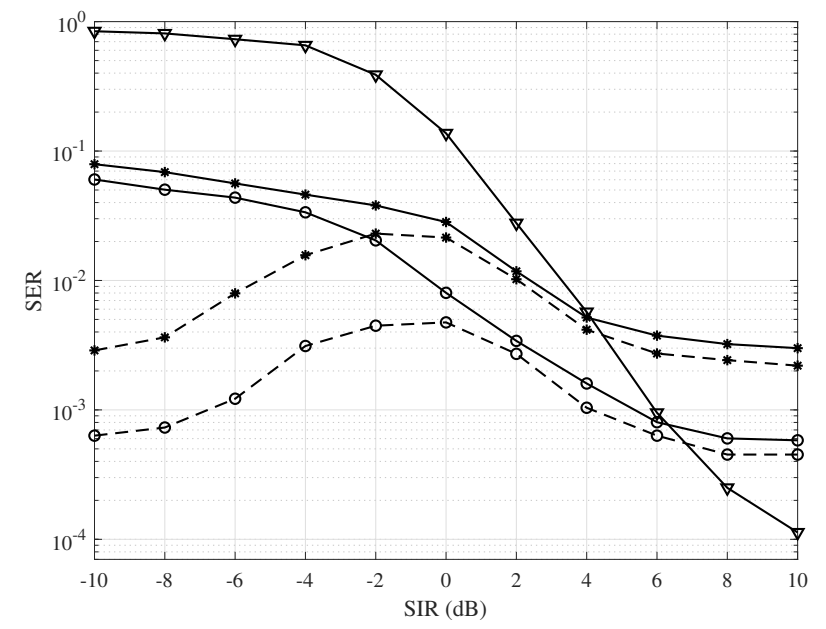

Fig. 7: SER as a function of the SIR for the classical detector $[\nabla]$, the DFNN-based $[*]$, and the CNN-based [o]. The SNR for the selected user is set to $-6 \mathrm{~dB}$. Two different cases are considered: plots in solid line [-] networks trained for $\lambda=0.25$, plots in dashed line [--] networks trained for each SIR value.

strongest, the capture effect happens for all receivers while being improved for the CNN-based receiver when the SIR is under $6 \mathrm{~dB}$. However, when $\mathrm{SIR}<0 \mathrm{~dB}$, i.e., the interfering user is the strongest, performance can be further improved if we have information on the SIR, i.e., learning is performed given the SIR value. In fact, when the SIR is known, the deep learning-based approach makes it possible to distinguish between the two users based on their power level, unless there is ambiguity, i.e., $\operatorname{SIR}=0 \mathrm{~dB}$.

\section{Computational Complexity}

The computational complexity of the classical LoRa receiver is of $\mathcal{O}(M \log (M))$ [9]. For the DFNN-based receiver, the complexity results in $\mathcal{O}\left(M^{2}\right)$. Based on [10], where the complexity order for the convolutional layers is derived, the overall complexity of the CNN-based receiver is also of $\mathcal{O}\left(M^{2}\right)$. Even though the proposed receivers and the classical LoRa receiver have different complexity orders, the proposed schemes' running time remains polynomial. According to [11], one of the definitions for computational efficiency is the following: "an algorithm is efficient if it has a polynomial running time". Besides, considering that this work focuses on the uplink case, where the gateway is plugged into a power source, issues of battery life and autonomy that may result from high complexity are not of concern.

\section{CONCLUSION}

This article investigated a deep learning-based approach for LoRa-like networks to decode a selected user's signals while considering interfering users that transmit simultaneously over the same frequency channel with the same SF. From the simulation results, we observed that in terms of SER, the two proposed receivers, the DFNN-based and the CNN-based, outperform the classical LoRa decoder in the presence of interference. In the absence of interference, the $\mathrm{CNN}$-based receiver performs closely to the classical LoRa receiver, which is the optimal receiver for the interferencefree case. However, the DFNN-based receiver has endured a slight loss in performance, e.g., a $2 \mathrm{~dB}$ penalty in SNR for a target SER of $10^{-4}$. The good performance shown by the proposed schemes is achieved while keeping an efficient complexity order, i.e., a polynomial running time of order two.

For the future, the deep learning-based approach seems to be a promising candidate to tackle the issue of interference in LoRa networks due to the exponential growth of connected devices. In addition to improving the capture effect, i.e., decoding the useful user when its power is higher than that of the interference, we have shown that the deep-learning-based approach makes it possible to decode the useful user when it is weaker than the interference. This requires information on the SIR. As a perspective, an SIR estimation technique could be combined with the deep learning-based decoder to improve the receiver's performance further.

\section{REFERENCES}

[1] Q. M. Qadir, "Analysis of the reliability of LoRa," IEEE Communications Letters, pp. 1-1, 2020.

[2] P. Edward, S. Elzeiny, M. Ashour, and T. Elshabrawy, "On the coexistence of LoRa- and Interleaved Chirp Spreading LoRa-Based modulations," in 2019 International Conference on WiMob, 2019.

[3] A. A. Tesfay, E. P. Simon, G. Ferré, and L. Clavier, "Serial interference cancellation for improving uplink in LoRa-like networks," in 2020 IEEE 31st Annual International Symposium on PIMRC, London, UK, 2020.

[4] N. E. Rachkidy, A. Guitton, and M. Kaneko, "Decoding superposed LoRa signals," in 2018 IEEE 43rd Conference on Local Computer Networks (LCN), 2018, pp. 184-190.

[5] P. Zhang, X. Kang, D. Wu, and R. Wang, "High-Accuracy Entity State Prediction Method Based on Deep Belief Network Toward IoT Search," IEEE Wireless Communications Letters, vol. 8, no. 2, pp. 492-495, 2019.

[6] J. Zhou, Y. Wang, K. Ota, and M. Dong, "AAIoT: Accelerating Artificial Intelligence in IoT Systems," IEEE Wireless Communications Letters, vol. 8, no. 3, pp. 825-828, 2019.

[7] H. Ye, G. Y. Li, and B.-H. Juang, "Power of deep learning for channel estimation and signal detection in ofdm systems," IEEE Wireless Communications Letters, vol. 7, no. 1, pp. 114-117, 2017.

[8] B. Reynders and S. Pollin, "Chirp spread spectrum as a modulation technique for long range communication," in 2016 Symposium on Communications and Vehicular Technologies (SCVT), 2016, pp. 1-5.

[9] A. A. Tesfay, E. P. Simon, I. Nevat, and L. Clavier, "Multiuser detection for downlink communication in LoRa- Like networks," IEEE Access, vol. 8, pp. 199001-199015, 2020.

[10] K. He and J. Sun, "Convolutional Neural Networks at constrained time cost," in 2015 IEEE Conference on Computer Vision and Pattern Recognition (CVPR), 2015, pp. 5353-5360.

[11] J. Kleinberg and É. Tardos, Algorithm Design, 1st ed. Pearson Education, 2005, ch. Basics of Algorithm Analysis, pp. 30-35. 